\title{
ARTICLE
}

Chronic lymphocytic leukemia

\section{Obinutuzumab plus fludarabine and cyclophosphamide in previously untreated, fit patients with chronic lymphocytic leukemia: a subgroup analysis of the GREEN study}

\author{
Francesc Bosch $\mathbb{D}^{1}$. Guy Cantin ${ }^{2} \cdot$ Agostino Cortelezzi $^{3} \cdot$ Wolfgang Knauf $^{4} \cdot$ Mourad Tiab $^{5} \cdot$ Mehmet Turgut $^{6}$.

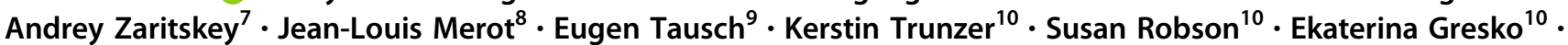 \\ Sebastian Böttcher ${ }^{11} \cdot$ Robin Foà $^{12} \cdot$ Stephan Stilgenbauer $^{9} \cdot$ Véronique Leblond $^{13}$
}

Received: 28 March 2019 / Revised: 21 June 2019 / Accepted: 4 July 2019 / Published online: 27 August 2019

(c) The Author(s) 2019. This article is published with open access

\begin{abstract}
GREEN (NCT01905943) is a nonrandomized, open-label, single-arm, phase 3b study investigating the safety and efficacy of obinutuzumab alone or in combination with chemotherapy in chronic lymphocytic leukemia (CLL). We report the preplanned subgroup analysis of 140 previously untreated, fit CLL patients who received obinutuzumab plus fludarabine and cyclophosphamide (G-FC). The primary endpoint was safety and tolerability. Efficacy was the secondary endpoint. Obinutuzumab $1000 \mathrm{mg}$ was administered intravenously on Day (D)1 (dose split D1-2), D8 and D15 of Cycle (C)1, and D1 of C2-6 (28-day cycles). Standard intravenous/oral doses of fludarabine and cyclophosphamide were administered on D1-3 of C1-6. Overall, $87.1 \%$ of patients experienced grade $\geq 3$ adverse events (AEs), including neutropenia (67.1\%) and thrombocytopenia (17.1\%). Serious AEs were experienced by $42.1 \%$ of patients. Rates of grade $\geq 3$ infusion-related reactions and infections were $19.3 \%$ and $15.7 \%$, respectively. Overall response rate was observed in $90.0 \%$, with $46.4 \%$ of patients achieving complete response (CR; including CR with incomplete marrow recovery). Minimal residual disease negativity rates were $64.3 \%$ in peripheral blood and $35.7 \%$ in bone marrow (intent-to-treat analysis). After a median observation time of 25.6 months, 2 year progression-free survival was $91 \%$. Frontline G-FC represents a promising treatment option for fit patients with CLL.
\end{abstract}

Supplementary information The online version of this article (https:// doi.org/10.1038/s41375-019-0554-1) contains supplementary material, which is available to authorized users.

Francesc Bosch

fbosch@vhio.net

1 University Hospital Vall d'Hebron, Barcelona, Spain

2 Hopital De L'Enfant—Jesus, Quebec City, QC, Canada

3 Hematology Unit, Policlinico Hospital and University of Milan, Milan, Italy

4 Onkologische Gemeinschaftspraxis, Agaplesion Bethanien Krankenhaus, Frankfurt, Germany

5 University Hospital, La Roche Sur Yon, France

6 Ondokuz Mayis University, Samsun, Turkey

7 Almazov National Medical Research Center, St Petersburg, Russia

\section{Introduction}

Chronic lymphocytic leukemia (CLL) is the most common leukemia in Western countries, with an annual, age-adjusted incidence of approximately four per 100,000 inhabitants $[1,2]$. Frontline treatment decisions in symptomatic patients

\section{IQVIA, St Ouen, France}

9 Department of Internal Medicine III, Ulm University, Ulm and Innere Medizin I, Universitätsklinikum des Saarlandes, Homburg, Germany

10 F. Hoffmann-La Roche Ltd, Basel, Switzerland

11 Second Department of Medicine, University of SchleswigHolstein, Campus Kiel, Kiel, Germany and Clinic III, Hematology, Oncology and Palliative Medicine, Rostock University Medical Center, Rostock, Germany

12 Hematology, Sapienza University, Rome, Italy

13 Sorbonne Université, AP-HP Hôpital Pitié Salpêtrière, Paris, France 
are generally based on age, physical fitness, and the presence of genetic risk factors, such as chromosome $17 \mathrm{p}$ deletion and TP53 gene mutation [1-4]. Although the therapeutic landscape in CLL has experienced a dramatic shift in recent years with the advent of new biologic agents, e.g., ibrutinib [5-10], chemoimmunotherapy remains a well-established frontline approach for a subset of patients with CLL [1, 11-19].

The combination of the anti-CD20 antibody, rituximab, plus fludarabine, and cyclophosphamide (R-FC) has been considered the standard of care for previously untreated, young, fit CLL patients who are eligible for this chemoimmunotherapy $[16,20]$. This regimen significantly improves progression-free survival (PFS) and overall survival (OS) compared with FC alone [11, 20-23], and provides a superior PFS benefit compared with rituximab plus bendamustine [12]. Higher rates of minimal residual disease (MRD) negativity are also achieved with R-FC compared with FC alone [24], and there is growing evidence that therapies capable of eliminating MRD lead to improved clinical outcome [24-31]. Although R-FC currently remains the standard of care, the therapeutic paradigm is constantly evolving. Recent interim analysis of ibrutinib in combination with rituximab (IR) has demonstrated superior efficacy and a favorable safety profile versus R-FC [32]. However, while a subset of CLL patients receiving R-FC or IR as frontline therapy experience durable remission rates $[11,23,32-36]$, the majority of patients are still destined to relapse. Obinutuzumab (GA101) is a glycoengineered type II anti-CD20 antibody that was developed in an attempt to improve therapeutic efficacy compared with rituximab. To date, obinutuzumab has demonstrated substantial activity in CLL [14, 17, 18, 37-39], and the additional benefit of obinutuzumab-based chemoimmunotherapy over rituximabbased chemoimmunotherapy has been observed in the pivotal phase 3 CLL11 trial [14, 18, 19]. In the CLL11 trial, obinutuzumab plus chlorambucil (G-Clb) provided a significant prolongation of PFS and OS in patients with CLL and comorbidities compared with rituximab plus chlorambucil (R-Clb) [14, 18, 19]. G-Clb also had an acceptable toxicity profile. While an increased risk of infusion-related reactions (IRRs) and neutropenia was observed, these adverse events (AEs) were manageable [14].

This superior efficacy of G-Clb over R-Clb, as seen in CLL11, has led to interest in evaluating the clinical activity of obinutuzumab in combination with other chemotherapies, such as bendamustine and FC [39, 40]. To date, frontline obinutuzumab plus bendamustine (G-B) has shown encouraging efficacy and manageable toxicity in patients with CLL [40]. Data from the phase $1 \mathrm{~b}$ feasibility study (GALTON) have shown that frontline G-FC is also tolerable and shows signs of clinical activity in CLL [39], but larger, confirmatory studies are needed.
GREEN (NCT01905943) is a phase 3b safety study of obinutuzumab alone or in combination with chemotherapy (investigator's choice) in 972 patients with previously untreated or relapsed/refractory CLL, including both fit and unfit patients. This paper reports the safety and efficacy results from a pre-specified subgroup of 140 previously untreated, fit patients who received G-FC in GREEN.

\section{Subjects and methods}

\section{Study design and patients}

GREEN is an international, multicenter phase $3 b$ safety study, with a nonrandomized, noncomparative, open-label design. The primary objective is to assess the safety and tolerability of obinutuzumab alone or in combination with various chemotherapy regimens. Chemotherapy options were partly dependent on patient fitness and based on investigator's choice. Assessment of efficacy is the secondary objective. In an attempt to mitigate the risk of IRRs, an exploratory objective of GREEN is to investigate alternative measures for obinutuzumab administration. A comparison of the IRR risk mitigation strategies employed in GREEN has been reported separately [41].

The inclusion criteria for patients in the G-FC subgroup included: aged $\geq 18$ years with previously untreated CLL requiring treatment per the International Workshop on CLL criteria [4]; adequate hematologic function (defined as hemoglobin $\geq 9.0 \mathrm{~g} / \mathrm{dL}$, an absolute neutrophil count of $\geq 1.5 \times 10^{9} / \mathrm{L}$, and platelets $\geq 75 \times 10^{9} / \mathrm{L}$ ); and an Eastern Cooperative Oncology Group performance status of $0-2$. All patients had to be fit (defined as a Cumulative Illness Rating Scale score of $\leq 6$ and a creatinine clearance $\geq 70 \mathrm{~mL} /$ min) to receive FC as chemotherapy. Patients with a $17 \mathrm{p}$ deletion and/or TP53 mutation could be included at the investigator's discretion [41].

Patients received obinutuzumab $1000 \mathrm{mg}$ intravenously (IV) on Day (D)1, 8, and 15 of Cycle (C)1, and on D1 of C2-6 for six 28-day cycles. To explore potential measures to reduce the risk of IRRs, the C1D1 dose of obinutuzumab was administered over two days, and patients were allocated to three cohorts: $25 \mathrm{mg}(12.5 \mathrm{mg} / \mathrm{h} ; \mathrm{C} 1 \mathrm{D} 1)+975 \mathrm{mg}$ (50-400 mg/h; C1D2) (Cohorts 1 and 3); $100 \mathrm{mg}(25 \mathrm{mg} / \mathrm{h}$; C1D1) + $900 \mathrm{mg}$ (50-400 mg/h; C1D2) (Cohort 2). All patients received prednisolone IV $100 \mathrm{mg}$ (or equivalent) $1 \mathrm{~h}$ pre dose on C1D1/D2, and patients in Cohorts 2 and 3 received additional corticosteroids (oral dexamethasone $20 \mathrm{mg}$ or equivalent dose of betamethasone $16-20 \mathrm{mg}$ ) $12 \mathrm{~h}$ before the first dose of obinutuzumab. FC were administered on D1-3 of each cycle at standard doses, either IV (fludarabine $25 \mathrm{mg} / \mathrm{m}^{2}$ over $30 \mathrm{~min}$ and cyclophosphamide 
$250 \mathrm{mg} / \mathrm{m}^{2}$ over $15-30 \mathrm{~min}$ ) or orally (fludarabine $40 \mathrm{mg} / \mathrm{m}^{2}$ and cyclophosphamide $250 \mathrm{mg} / \mathrm{m}^{2}$ ).

Measures to minimize the risk of tumor lysis syndrome (TLS) in patients with high tumor burden (peripheral blood lymphocyte count $\geq 25 \times 10^{9} / \mathrm{L}$ or bulky lymphadenopathy) were also applied; these included appropriate hydration, pretreatment with allopurinol or a suitable alternative, intensive laboratory monitoring, implementation of an expanded risk definition, investigator training, and patient education [40]. Use of granulocyte-colony stimulating factor was permitted, with primary prophylaxis recommended for patients aged $\geq 60$ years and/or with comorbidities. Antimicrobial prophylaxis was not mandated by the study protocol in GREEN but could be given at the investigator's discretion.

GREEN was conducted in accordance with the Declaration of Helsinki, Good Clinical Practice guidelines, and all applicable local laws and regulations. The study protocol and its amendments, and other study-related materials were approved by institutional review boards/ ethics committees at participating centers. Written informed consent was provided by all patients.

\section{Study endpoints}

The primary outcome measure was safety, including AEs, grade $\geq 3$ AEs, serious AEs, AEs of special interest (AESI; IRRs, infections, neutropenia, and TLS), AEs of particular interest (AEPI; including thrombocytopenia, second malignancies, hepatitis $\mathrm{B}$ reactivation, and hemorrhagic events), dose delays/discontinuations, and laboratory abnormalities.

Secondary efficacy endpoints included overall response rate (ORR; defined as a confirmed complete response [CR] plus CR with incomplete marrow recovery [CRi], or partial response [PR], as determined by the study investigator, at the time of the final response assessment), duration of response (defined as the period from the date of initial confirmed PR or CR until the date of progressive disease or death from any cause), PFS (defined as the time from the date of treatment initiation until the first documented disease progression or death from any cause), time to new antileukemic therapy (defined as the time between the date of treatment initiation and first intake of new antileukemic therapy), and MRD negativity rate (defined as $<1$ CLL cell per 10,000 leukocytes) in peripheral blood or bone marrow.

\section{Assessments}

AEs, serious AEs, and AESI/AEPI were monitored, and graded according to the National Cancer Institute's Common Terminology Criteria for AEs version 4.0. IRRs were defined as any $\mathrm{AE}$ that occurred during or within $24 \mathrm{~h}$ of obinutuzumab infusion and were considered related to obinutuzumab. Other safety assessments included physical examinations, electrocardiogram, vital signs, and laboratory parameters.

Tumor response was assessed by investigators according to International Workshop on CLL criteria [4] at the final response assessment, which occurred $\sim 3$ months after the last dose of study treatment. A computed tomography scan was required to confirm $\mathrm{CR}$ and $\mathrm{PR}$. A bone marrow biopsy was required for confirmation of $\mathrm{CR}$. Patients lacking a valid biopsy, but who otherwise met $\mathrm{CR}$ criteria, were classed by the investigator as PR.

MRD was assessed in peripheral blood (all patients) or bone marrow (in patients requiring confirmation of $\mathrm{CR}$ ), also at the final response assessment; analysis was by fourcolor flow cytometry, undertaken in a EuroFlow-certified central laboratory in Kiel, Germany [42, 43].

\section{Statistical methodology}

There was no hypothesis testing in GREEN and therefore no power calculation. The sample size estimation of 950 patients for the overall study population was based on the ability to detect nonfrequent AEs (irrespective of grade) in order to provide insight into the overall safety profile of obinutuzumab alone or with chemotherapy. This is a prespecified subgroup analysis of fit patients treated with G-FC in the GREEN study.

The safety population comprised patients who received $\geq 1$ dose of study treatment and was used for all safety analyses. Safety data are presented using descriptive statistics. Efficacy endpoints were assessed in all patients regardless of whether they received any therapy (intent-totreat [ITT] population). For the MRD analyses, the intentto-ship population comprised all patients whose MRD samples at the final response assessment could be shipped to the central laboratory within $48 \mathrm{~h}$; centers unable to supply fresh samples to the central laboratory within $48 \mathrm{~h}$ of sampling were excluded from the MRD analyses. The MRD-evaluable population comprised all patients within the intent-to-ship population with an evaluable MRD result (peripheral blood or bone marrow).

The ORR, CR rate, and MRD negativity rate are presented with two-sided 95\% Clopper-Pearson confidence intervals (CIs). Time-to-event endpoints were estimated using Kaplan-Meier methodology.

The analysis presented here formed part of the primary analysis and took place after all patients had undergone the final response assessment, which occurred $\sim 3$ months after completion of study treatment (data cutoff, December 29, 2016). After the data snapshot was taken for analysis, a few additional AEs (seven AEs in six patients) were reported late by sites on the database, which remained open to 
continue collecting information until the final analysis (Supplementary Table S1). In addition, one patient with CR at the final response assessment had this changed to CRi (Supplementary Table S2). These updates are not part of the statistical analysis or summary tables that are presented.

\section{Results}

\section{Patients}

Patients (including those reported in this subgroup analysis) were enrolled between October 2013 and March 2016 at 195 centers in 31 countries in Africa, North and South America, Asia, and Europe. Both the ITT and safety populations in this analysis comprised 140 fit patients with previously untreated CLL; all patients received G-FC treatment. At the time of analysis, 11 patients had discontinued the study, and 129 were still ongoing (all in follow-up). Primary reasons for study discontinuation were death $(n=4)$, withdrawal of consent $(n=4), \operatorname{AE}(n=1$; IRR: cytokine release syndrome), investigator decision $(n=1)$, and other $(n=1)$.

Median age was 57 (range, 34-74) years; $67.9 \%$ of patients were male, and most had advanced Binet stage at screening (Table 1). IGHV was mutated in $26.4 \%$ of patients, unmutated in $55.7 \%$, and missing in $17.9 \%$. Chromosomal abnormalities detected by fluorescence in situ hybridization were present in $75.7 \%$ of patients, $55.7 \%$ were ZAP70 positive, and $40.7 \%$ were CD38 positive.

\section{Treatment exposure}

In total, 124 patients (88.6\%) completed all study treatment per protocol. Reasons for not completing study treatment were tolerability/AEs $(n=14)$, investigator's decision $(n=$ 1 ), and withdrawal of consent ( $n=1)$ (Fig. 1).

Mean number of obinutuzumab administrations was 8.8 (planned, 9), with 95.0\% $(n=133)$ of patients receiving $\geq 90 \%$ of the planned dose. Median obinutuzumab exposure time was 21.1 (range, 0.3-28.1) weeks. Mean number of chemotherapy cycles administered was 5.7 for both cyclophosphamide and fludarabine (planned, 6), with $94.3 \%$ of patients $(n=132)$ receiving $\geq 90 \%$ of the planned doses of both drugs. In total, 104 patients $(74.3 \%)$ received previous or concomitant granulocyte-colony stimulating factor administration.

\section{Safety}

Median observation time was 25.6 (range, 2.0-37.8) months. In the safety analysis, most patients $(98.6 \% ; n=$ 138) reported $\geq 1 \mathrm{AE}$ of any grade. The most frequently
Table 1 Baseline demographics and disease characteristics (G-FC population, previously untreated, fit)

\begin{tabular}{|c|c|}
\hline Characteristic & All patients $(n=140)$ \\
\hline Median age, years (range) & $57(34-74)$ \\
\hline Male, $n(\%)$ & $95(67.9)$ \\
\hline Median CIRS score (range) & $2(0-6)$ \\
\hline \multicolumn{2}{|l|}{ ECOG performance status, $n(\%)$} \\
\hline 0 & $108(77.1)$ \\
\hline 1 & $32(22.9)$ \\
\hline \multicolumn{2}{|l|}{ Binet stage at screening, $n(\%)$} \\
\hline A & $37(26.4)$ \\
\hline B & $74(52.9)$ \\
\hline $\mathrm{C}$ & $29(20.7)$ \\
\hline $\begin{array}{l}\text { Absolute lymphocyte count, } n(\%) \geq 50 \times \\
10^{9} / \mathrm{L}\end{array}$ & $93(66.4)$ \\
\hline Tumor bulk $\geq 5 \mathrm{~cm}, n(\%)$ & $101(72.1)$ \\
\hline \multicolumn{2}{|l|}{ Genomic aberrations, $n(\%)^{*}$} \\
\hline $17 \mathrm{p}$ deletion & $4(2.9)$ \\
\hline 11q deletion & $30(21.4)$ \\
\hline $12 q$ trisomy & $20(14.3)$ \\
\hline $13 q$ deletion & $43(30.7)$ \\
\hline Other aberrations & $9(6.4)$ \\
\hline No abnormality & $18(12.9)$ \\
\hline Missing & $16(11.4)$ \\
\hline \multicolumn{2}{|l|}{ IGHV, $n(\%)$} \\
\hline Unmutated & $78(55.7)$ \\
\hline Mutated & $37(26.4)$ \\
\hline Missing & $25(17.9)$ \\
\hline \multicolumn{2}{|l|}{ ZAP70, $n(\%)$} \\
\hline Positive & $78(55.7)$ \\
\hline Negative & $40(28.6)$ \\
\hline Missing $^{\dagger}$ & $22(15.7)$ \\
\hline \multicolumn{2}{|l|}{$\mathrm{CD} 38, n(\%)$} \\
\hline Positive & $57(40.7)$ \\
\hline Negative & $60(42.9)$ \\
\hline Missing ${ }^{\ddagger}$ & $23(16.4)$ \\
\hline
\end{tabular}

CIRS Cumulative Illness Rating Scale, $\mathrm{CrCl}$ creatinine clearance, ECOG Eastern Cooperative Oncology Group, $G-F C$ obinutuzumab plus fludarabine and cyclophosphamide, $I G H V$ immunoglobulin heavy chain variable region

*According to the hierarchical model of genomic aberrations

$\dagger 22$ patients had missing central lab evaluation, of whom 6 had local lab assessment ( 3 positive, 3 negative) and the remaining 16 patients had missing local lab evaluation

$\$ 7$ patients had local lab assessment (5 positive, 2 negative)

reported treatment-emergent AEs of any grade (by preferred term, affecting $\geq 20 \%$ of patients) were neutropenia (75.7\%), nausea $(42.1 \%)$, pyrexia $(37.1 \%)$, thrombocytopenia (35.0\%), anemia (25.7\%), vomiting $(25.7 \%)$, and diarrhea $(20.7 \%)$. AEs were considered related to treatment in $95.7 \%$ 
Fig. 1 Patient flow diagram. *Comprised all patients within the intent-to-ship population with an evaluable MRD result (peripheral blood or bone marrow); ‘still ongoing. $\mathrm{AE}$ adverse event, G-FC obinutuzumab plus fludarabine and cyclophosphamide, ITT intent-to-treat, MRD minimal residual disease

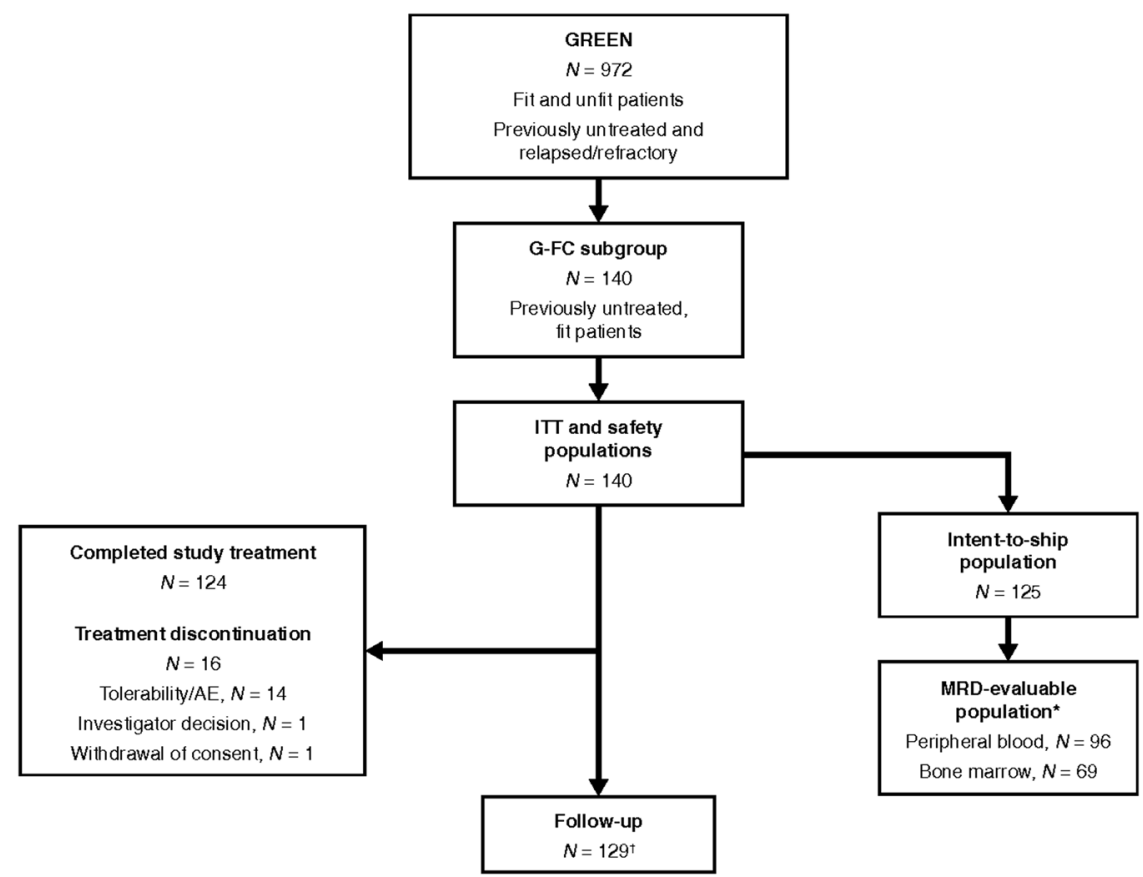

of patients, most commonly neutropenia (72.9\%), nausea $(38.6 \%)$, thrombocytopenia $(32.9 \%)$, pyrexia $(28.6 \%)$, vomiting $(21.4 \%)$, and anemia $(20.7 \%)$.

In total, $87.1 \%$ of patients experienced grade $\geq 3$ AEs (Table 2), the most common of which (affecting $>5 \%$ of patients) were neutropenia $(67.1 \%)$, thrombocytopenia $(17.1 \%)$, anemia $(10.7 \%)$, leukopenia $(7.9 \%)$, febrile neutropenia $(7.1 \%)$, and pneumonia $(5.7 \%)$. Serious AEs were experienced by $42.1 \%$ of patients (Table 2), and included neutropenia $(15.7 \%)$, febrile neutropenia $(6.4 \%)$, pneumonia $(5.7 \%)$, and pyrexia $(5.7 \%)$.

AESI/AEPI (basket terms) included neutropenia (any grade, $77.9 \%$, grade $\geq 3,70.7 \%$ ), IRRs (any grade, $69.3 \%$; grade $\geq 3,19.3 \%$ [Supplementary Table S3]), infections (any grade, 56.4\%, grade $\geq 3,15.7 \%$ ), thrombocytopenia (any grade, $37.1 \%$, grade $\geq 3,17.9 \%$ ), second malignancies according to Medical Dictionary for Regulatory Activities (MedDRA) system organ class (any grade, $4.3 \%$; grade $\geq 3$, $3.6 \%$ ), and TLS (any grade, 2.1\%; all grade 3, and all laboratory TLS) (Table 2). Sixty patients $(55.0 \%)$ required a modified treatment regimen (obinutuzumab or FC) due to neutropenia (reported as an AESI/AEPI). The most common infections (affecting $\geq 5 \%$ of patients) by preferred term were bronchitis $(8.6 \%)$, pneumonia $(7.1 \%)$, nasopharyngitis $(7.1 \%)$, upper respiratory tract infection $(6.4 \%)$, influenza $(5.7 \%)$, sinusitis $(5.7 \%)$, and herpes zoster $(5.0 \%)$. The majority of infections were bacterial; there were no cases of pneumocystis pneumonia infection, and one case each of candida and fungal infection. Opportunistic infections included two cases of herpes simplex virus infection, and one case each of varicella zoster virus, listeriosis, and lymph node tuberculosis infection.

Fourteen patients $(10.0 \%)$ discontinued G-FC treatment prematurely because of AEs, most common AESI/AEPI were neutropenia $(3.6 \% ; n=5)$, thrombocytopenia $(2.1 \%$; $n=3)$, or infections $(1.4 \% ; n=2)$. There were four AEs leading to death (one case each of sepsis, second malignancy [acute myeloid leukemia], pneumonia, and unexplained death) (Supplementary Table S4); three of the four patients who died had previously discontinued treatment due to AEs. One additional patient died due to PD.

\section{Response rate at final response assessment}

ORR at the final response assessment was $90.0 \%$ (95\% CI: 83.8-94.4), with $46.4 \%$ of patients achieving a CR (including CRi) and $43.6 \%$ of patients achieving a PR (Table 3). Response was missing or not evaluable in eleven patients $(7.9 \%)$. Of the four patients with $17 \mathrm{p}$ deletions, one achieved $\mathrm{CR}$ and one had progressive disease (response not available in two patients).

\section{MRD negativity rate at final response assessment}

For the ITT population, MRD negativity rates were $64.3 \%$ $(90 / 140)$ and $35.7 \%(50 / 140)$ in peripheral blood and bone marrow, respectively (Table 4). MRD negativity rates in the intent-to-ship population, comprising 125 patients, were $72.0 \%(90 / 125)$ in peripheral blood and $40.0 \%(50 / 125)$ in bone marrow. The MRD-evaluable population comprised 
Table 2 Safety overview (safety population)

\begin{tabular}{|c|c|}
\hline & $\begin{array}{l}\text { Number }(\%) \text { of patients } \\
\text { reporting AEs }(n=140)\end{array}$ \\
\hline \multicolumn{2}{|c|}{ Grade $\geq 3$ AEs by preferred term (reported by $\geq 2 \%$ of patients) } \\
\hline Any & $122(87.1)$ \\
\hline Neutropenia & $94(67.1)$ \\
\hline Thrombocytopenia & $24(17.1)$ \\
\hline Anemia & 15 (10.7) \\
\hline Leukopenia & $11(7.9)$ \\
\hline Febrile neutropenia & $10(7.1)$ \\
\hline Pneumonia & $8(5.7)$ \\
\hline Lymphopenia & $7(5.0)$ \\
\hline Hypertension & $4(2.9)$ \\
\hline Hyperglycemia & $4(2.9)$ \\
\hline Neutrophil count decreased & $4(2.9)$ \\
\hline TLS & $3(2.1)$ \\
\hline Hypotension & $3(2.1)$ \\
\hline Lung infection & $3(2.1)$ \\
\hline Pyrexia & $3(2.1)$ \\
\hline \multicolumn{2}{|c|}{ SAEs by preferred term (reported by $\geq 2 \%$ of patients) } \\
\hline Any & $59(42.1)$ \\
\hline Neutropenia & $22(15.7)$ \\
\hline Febrile neutropenia & $9(6.4)$ \\
\hline Pneumonia & $8(5.7)$ \\
\hline Pyrexia & $8(5.7)$ \\
\hline Thrombocytopenia & $4(2.9)$ \\
\hline Anemia & $3(2.1)$ \\
\hline Lung infection & $3(2.1)$ \\
\hline \multicolumn{2}{|l|}{ Grade $\geq 3$ AESI/AEPI (basket terms) } \\
\hline Neutropenia* & 99 (70.7) \\
\hline $\operatorname{IRRs}^{\dagger}$ & $27(19.3)$ \\
\hline Thrombocytopenia ${ }^{\ddagger}$ & $25(17.9)$ \\
\hline Infections ${ }^{\S}$ & $22(15.7)$ \\
\hline Second malignancies by MedDRA SOC ${ }^{\mathrm{I}}$ & $5(3.6)$ \\
\hline TLS** & $3(2.1)$ \\
\hline Hepatitis $\mathrm{B}$ reactivation $^{\dagger \dagger}$ & 0 \\
\hline Hemorrhagic events & 0 \\
\hline
\end{tabular}

$A E s$ adverse events, $A E P I$ adverse events of particular interest, $A E S I$ adverse events of special interest, IRR infusion-related reaction, MedDRA Medical Dictionary for Regulatory Activities, $S A E$ serious adverse event, $S M Q$ standardized MedDRA queries, SOC system organ class, $T L S$ tumor lysis syndrome

*Includes neutropenia, febrile neutropenia, neutrophil count decreased, and neutropenic sepsis

${ }^{\dagger}$ AEs that occurred during or within $24 \mathrm{~h}$ of obinutuzumab infusion and considered related to obinutuzumab

${ }^{\sharp}$ Includes thrombocytopenia and platelet count decreased

${ }^{\S}$ All AEs classified as infections and infestations (MedDRA SOC)

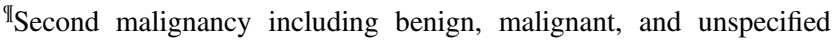
tumors (MedDRA SOC) occurring $>6$ months after first study drug intake

**Any AE with the preferred term "TLS"; one patient was classified as having both TLS and an IRR

${ }^{\dagger}$ Any AE with the preferred term containing "hepatitis B" or "hepatitis acute" that was additionally assessed as hepatitis B virus reactivation via medical review

$\$$ Includes hematoma, epistaxis, hematuria, conjunctival hemorrhage, ecchymosis, hematochezia, hematospermia, and metrorrhagia
Table 3 Response rates at the final response assessment (investigator assessment; ITT population)

\begin{tabular}{lc}
\hline$n(\%)[95 \% \mathrm{CI}]$ & All patients $(n=140)$ \\
\hline ORR & $126(90.0)[83.8-94.4]$ \\
CR & $37(26.4)[19.3-34.5]$ \\
CRi & $28(20.0)[13.7-27.6]$ \\
PR & $61(43.6)[35.2-52.2]$ \\
SD & $1(0.7)[0.0-3.9]$ \\
PD & $2(1.4)[0.2-5.1]$ \\
Missing or not evaluable & $11(7.9)$ \\
\hline
\end{tabular}

$C I$ confidence interval, $C R$ complete response, $C R i$ complete response with incomplete marrow recovery, ITT intent-to-treat, $O R R$ overall response rate, $P D$ progressive disease, $P R$ partial response, $S D$ stable disease

Table 4 MRD negativity rates and overall response in MRD-negative patients at the final response assessment

\begin{tabular}{ll}
\hline$n / N(\%)$ & All patients $(n=140)$ \\
\hline Peripheral blood & \\
Intent-to-treat population & $n=140$ \\
MRD negative & $90 / 140(64.3)$ \\
Intent-to-ship population & $n=125$ \\
MRD negative & $90 / 125(72.0)$ \\
Evaluable population & $n=96$ \\
MRD negative & $90 / 96(93.8)$ \\
CR & $26 / 90(28.9)$ \\
CRi & $19 / 90(21.1)$ \\
PR & $41 / 90(45.6)$ \\
SD & $1 / 90(1.1)$ \\
Missing & 3 \\
Bone marrow & \\
Intent-to-treat population & $n=140$ \\
MRD negative & $50 / 140(35.7)$ \\
Intent-to-ship population & $n=125$ \\
MRD negative & $50 / 125(40.0)$ \\
Evaluable population & $n=69$ \\
MRD negative & $50 / 69(72.5)$
\end{tabular}

$C R$ complete response, $C R i$ complete response with incomplete marrow recovery, $G-F C$ obinutuzumab plus fludarabine and cyclophosphamide, $M R D$ minimal residual disease, $P R$ partial response, $S D$ stable disease

${ }^{a}$ Bone marrow samples were only collected from patients with suspected $\mathrm{CR}$ or CRi at the final response assessment

96 patients with an evaluable peripheral blood sample and 69 patients with a bone marrow sample. MRD negativity rates in the MRD-evaluable population were 93.8\% (90/96) and $72.5 \%$ (50/69) for peripheral blood and bone marrow, respectively. 
Among IGHV mutated patients $(n=37)$ with an evaluable peripheral blood $(n=29)$ and bone marrow sample $(n=21)$, MRD negativity rates were $96.6 \%(28 / 29)$ and $66.7 \%(14 / 21)$, respectively. In IGHV unmutated patients $(n=77)$, MRD negativity rates were $91.5 \%(54 / 59)$ and $72.1 \%(31 / 43)$ in peripheral blood and bone marrow, respectively (Supplementary Table S5).

\section{Progression-free survival and time to new antileukemic therapy}

After a median observation time of 25.6 (range, 2.0-37.8) months and 12 PFS events, median PFS was not reached (Fig. 2). At 2 years, estimated PFS was 91\% (95\% CI: 84$96 \%)$. Among IGHV mutated $(n=37)$ and unmutated $(n=$ $78)$ patients, 2 year PFS was $96 \%$ (95\% CI: 75-99\%) and $88 \%$ (95\% CI: 76-94\%), respectively (Supplementary Fig. S1). Six patients received new antileukemia therapy, with time to new antileukemic therapy in these patients ranging from 1.2 to 37.8 months.

A meaningful analysis of PFS according to MRD response was not possible because of the small number MRD-positive patients ( $n=5$ in peripheral blood) and bias introduced by withdrawn patients not undergoing MRD evaluation at the final response assessment.

\section{Discussion}

The safety and efficacy of frontline G-FC was assessed in 140 fit CLL patients. Results from this subgroup analysis of the international, phase $3 \mathrm{~b}$ GREEN study largely support the preliminary findings of the phase $1 \mathrm{~b}$ GALTON study [39], and suggest that G-FC may have manageable toxicity and promising efficacy in this patient population. Strengths and limitations of the GREEN study have been described previously [41].

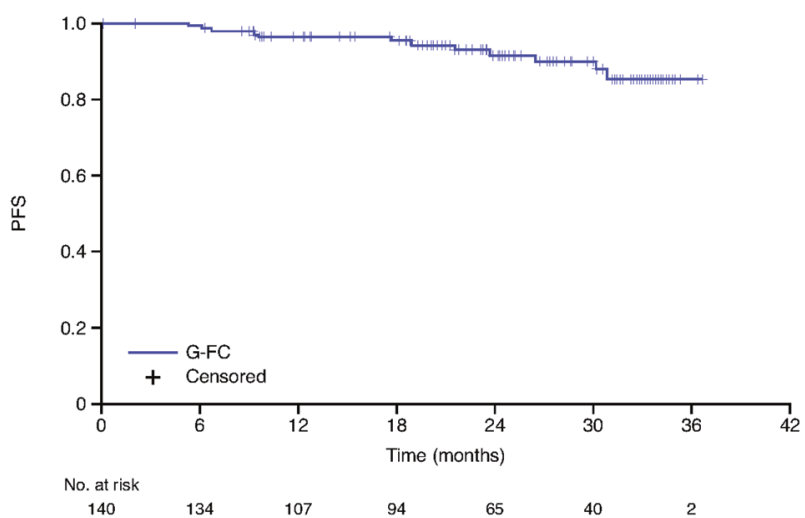

Fig. 2 Kaplan-Meier plot of progression-free survival (ITT population). G-FC obinutuzumab plus fludarabine and cyclophosphamide, ITT intent-to-treat, PFS progression-free survival
AEs reported in this study were clinically relevant but were consistent with the known safety profile of obinutuzumab [14, 17, 18, 37-39], with the most common being hematologic AEs, IRRs, and infections. The high rate of some AEs, particularly hematologic events, reflects the intensity of this treatment regimen; however, most patients could tolerate treatment, with only $10.0 \%$ discontinuing G-FC due to AEs. No unexpected safety signals were observed, although the occurrence of IRRs, including TLS, highlights the need for careful risk assessment, prophylaxis, and monitoring of these events.

The rate of grade $\geq 3$ neutropenia in this analysis was $67.1 \%$. Among fit patients receiving frontline R-FC, incidence of grade $\geq 3$ neutropenia was $84.2 \%$ in the CLL10 study [12] and 34\% (grade 3-4) in the CLL8 study [20]. Some of the differences between studies can be accounted for by differences in safety observation times. Importantly, the relatively high rate of grade $\geq 3$ neutropenia $(70.7 \%)$ in the current analysis did not translate into a high rate of infection (15.7\%) [20]. The percentage of patients who experienced grade $\geq 3$ neutropenia was higher than that seen with the combination of G-B (48.6\% [fit patients only]) in the GREEN study [40], which is in accordance with the finding that R-B is associated with a lower incidence of neutropenia than R-FC, as reported in CLL10 [12].

Grade $\geq 3$ IRRs were experienced in $19.3 \%$ of patients, and most occurred during the first infusion. Of note, this rate of grade $\geq 3$ IRRs is higher than that generally seen for patients receiving rituximab-based chemoimmunotherapy; this may be owing to the greater level of B-cell depletion and accompanying proinflammatory cytokine release achieved with obinutuzumab compared with rituximab [44]. In other reports of obinutuzumab-based chemoimmunotherapy, grade $\geq 3$ IRRs were observed in $20 \%$ of patients treated with G-Clb in CLL11 [14] and in $17.1 \%$ of patients receiving G-B in Cohort 1 of GREEN [40]. No IRRs were fatal in this analysis.

In total, four patients died $(2.9 \%)$ due to an $\mathrm{AE}$ and 1 $(0.7 \%)$ patient died due to progression of disease. In comparison, incidence of treatment-related deaths with R-FC was $2.0 \%$ in CLL8 [20] and 5\% in CLL10 [12].

ORR $(90.0 \%)$, CR rate $(46.4 \%$, including CRi), and PFS rate ( $91 \%$ at 2 years) were favorable, suggesting that G-FC is clinically active in this setting. These findings are consistent with other studies, which have demonstrated the effectiveness of frontline immunochemotherapy in CLL, particularly in patients with a good biologic profile [11-13, 20-23, 40]. A high rate of MRD negativity was also achieved with G-FC (64.3\% in peripheral blood $[72.0 \%$ of the intent-to-ship and $93.8 \%$ of the MRD-evaluable population] and $35.7 \%$ in bone marrow [40.0\% of the intent-to-ship and $72.5 \%$ of the MRD-evaluable population]), which is particularly encouraging given the 
increasing evidence linking posttreatment MRD status with therapeutic outcome [24-31]. The discrepancy between the $\mathrm{CR}$ and MRD negativity rates in the present study is, however, currently unclear. In CLL10, the MRD negativity rate reported for fit CLL patients receiving frontline R-FC was $74 \%$ in peripheral blood and $58 \%$ in bone marrow (in the MRD-evaluable population only) [12]. Interestingly, the level of MRD negativity achieved with G-FC is only slightly higher than that achieved with G-B in the GREEN study (despite unfit and fit patients receiving G-B), although meaningful comparisons between regimens cannot be made due to the nonrandomized allocation of chemotherapy (a limitation of the study) and differences in sample size [40].

Nowadays, MRD can reliably be detected to a level of one CLL cell in 10,000 leukocytes. Although not routinely performed in clinical practice, MRD assessment has been included in many clinical trials using the flow cytometry technique harmonized and validated by the European Research Initiative on CLL [35, 38, 39]. Several of these trials have reported that MRD negativity is independently associated with longer PFS and OS in CLL patients after frontline therapy [24-31]. Recently, MRD has been identified as a potential surrogate marker for PFS [45], suggesting it could provide an early indication of efficacy. Such findings highlight the importance of utilizing MRDnegative remission as a standard endpoint in clinical trials.

Estimated 2 year PFS rates were higher for IGHV mutated patients $(96 \%)$ treated with G-FC compared with IGHV unmutated patients $(88 \%)$, while MRD negativity rates were comparable. This latter result appears to show high MRD response rates with G-FC regardless of IGHV mutation status, but this may also be partly due to biasing the results of MRD in peripheral blood within the intent-toship population $(n=125)$, as only a subset of these patients were MRD-evaluable $(n=96)$. Results from three separate studies suggest that CLL patients with mutated IGHV benefit substantially from frontline R-FC chemoimmunotherapy, and may even be cured with this regimen $[11,23,35]$.

Baseline characteristics were generally consistent with "typical" frontline, fit patients with CLL. The incidence of 17 p deletions (2.9\%) was low (typically 5-8\%) [36], likely reflecting an increasing move towards risk-adapted therapy. Patients with $17 \mathrm{p}$ deletions show a marked resistance to chemotherapy, which cannot be overcome by the addition of anti-CD20 antibodies [2, 20]. Instead, these patients should ideally be treated with novel agents like ibrutinib, unless contraindications suggest otherwise [2].

Ibrutinib has emerged as a well-tolerated and highly effective treatment option for CLL patients in the relapse setting, and there is a growing consensus in favor of frontline therapy with ibrutinib in elderly CLL patients and in CLL patients with poor prognostic factors [6-8]. It also has potential to be used in combination with rituximab and/ or immunochemotherapy in fit patients [32]. However, while ibrutinib may be associated with good outcomes and a favorable toxicity profile [6], long-term AEs and the impact of its cost on the health care systems remain to be elucidated $[46,47]$. There is also a need to better understand the reasons behind the high discontinuation rates reported for ibrutinib in real-life studies [48, 49].

In conclusion, G-FC represents a promising treatment option for young, fit patients with previously untreated CLL who are eligible for potent chemoimmunotherapy. Moreover, G-FC could hold potential as an effective "backbone" of combination therapies with new compounds, such as ibrutinib; such a regimen (G-FC plus ibrutinib) is already showing favorable efficacy in a phase 2 trial as first-line treatment for fit CLL patients with mutated IGHV [50]. No unexpected safety signals were observed with G-FC in the present study and side effects were generally manageable, although the high occurrence of neutropenia and IRRs, including TLS, highlights the need for careful risk assessment, prophylaxis, and monitoring of these events. The low rate of progression and high rate of MRD negativity indicate that G-FC is a clinically active treatment option for fit patients with CLL, although longer follow-up is required to confirm these results.

\section{Data availability}

"Qualified researchers may request access to individual patient level data through the clinical study data request platform (www.clinicalstudydatarequest.com). Further details on Roche's criteria for eligible studies are available here (https://clinicalstudydatarequest.com/Study-Sponsors/ Study-Sponsors-Roche.aspx). For further details on Roche's Global Policy on the Sharing of Clinical Information and how to request access to related clinical study documents, see here (https://www.roche.com/research_and_ development/who_we_are_how_we_work/clinical_trials/ our_commitment_to_data_sharing.htm)"

Acknowledgements The authors would like to thank the GREEN study investigators, coordinators, nurses, and patients. The authors would also like to thank Sara Prada for her valuable contribution to the statistical analyses. GREEN is sponsored by F. Hoffmann-La Roche Ltd. Third-party medical writing assistance was provided by Janis Noonan of Gardiner-Caldwell Communications, and was funded by F. Hoffmann-La Roche Ltd.

Funding This study was sponsored by F. Hoffmann-La Roche Ltd.

\section{Compliance with ethical standards}

Conflict of interest FB reports: consultancy, honoraria, and speakers bureau for Roche, Novartis, Janssen, Abbvie, Gilead, and Mundipharma; research funding from Roche, Celgene, Karyospharm, and Takeda; AZ 
reports: consultancy for Novartis and Janssen; speakers bureau for Novartis; J-LM and ET reports: research funding from Roche; KT reports: employment and equity ownership for Roche; SR reports: employment for Roche; EG reports: former employment for Roche; SB reports consulting fees from Roche and AbbVie; research funding from Roche, AbbVie, Janssen, and Celgene; and honoraria from Roche, AbbVie, Novartis, Janssen, and Becton Dickinson; SS reports: honoraria, consultancy, advisory board membership, travel support, research funding, and speakers bureau for AbbVie, Amgen, Celgene, Genentech, Gilead, GlaxoSmithKline, Janssen, Novartis, Pharmacyclics, and Roche; RF reports: consultancy and speakers bureau for Roche, Janssen, Gilead, Amgen, Celgene, BMS, Sandoz, Novartis, and Abbvie; VL reports: honoraria for Abbvie, Roche, Gilead, Janssen, BMS, Novartis, and Servier; advisory board membership for Abbvie, Roche, Gilead, Janssen, Novartis, and Servier; speakers bureau for Abbvie and Janssen; MT reports: advisory board membership, speaker bureau, and research funding from Roche; WK reports honoraria from Roche; GC, AC, and MT report no conflicts of interest.

Publisher's note: Springer Nature remains neutral with regard to jurisdictional claims in published maps and institutional affiliations.

Open Access This article is licensed under a Creative Commons Attribution 4.0 International License, which permits use, sharing, adaptation, distribution and reproduction in any medium or format, as long as you give appropriate credit to the original author(s) and the source, provide a link to the Creative Commons license, and indicate if changes were made. The images or other third party material in this article are included in the article's Creative Commons license, unless indicated otherwise in a credit line to the material. If material is not included in the article's Creative Commons license and your intended use is not permitted by statutory regulation or exceeds the permitted use, you will need to obtain permission directly from the copyright holder. To view a copy of this license, visit http://creativecommons. org/licenses/by/4.0/.

\section{References}

1. Eichhorst B, Robak T, Montserrat E, Ghia P, Hillmen P, Hallek M, et al. Chronic lymphocytic leukaemia: ESMO Clinical Practice Guidelines for diagnosis, treatment and follow-up. Ann Oncol. 2015;26(suppl 5):v78-v84.

2. Hallek M. Chronic lymphocytic leukemia: 2017 update on diagnosis, risk stratification, and treatment. Am J Hematol. 2017;92:946-65.

3. Stilgenbauer S. Prognostic markers and standard management of chronic lymphocytic leukemia. Hematol Am Soc Hematol Educ Program. 2015;2015:368-77.

4. Hallek M, Cheson BD, Catovsky D, Caligaris-Cappio F, Dighiero $\mathrm{G}$, Döhner H, et al. Guidelines for the diagnosis and treatment of chronic lymphocytic leukemia: a report from the International Workshop on Chronic Lymphocytic Leukemia updating the National Cancer Institute-Working Group 1996 guidelines. Blood. 2008;111:5446-56.

5. Byrd JC, Furman RR, Coutre SE, Burger JA, Blum KA, Coleman $\mathrm{M}$, et al. Three-year follow-up of treatment-naive and previously treated patients with CLL and SLL receiving single-agent ibrutinib. Blood. 2015;125:2497-506.

6. O'Brien S, Furman RR, Coutre S, Flinn IW, Burger JA, Blum K, et al. Single-agent ibrutinib in treatment-naive and relapsed/ refractory chronic lymphocytic leukemia: a 5-year experience. Blood. 2018;131:1910-9.

7. Barr P. Updated efficacy and safety from the phase 3 resonate- 2 study: Ibrutinib as first-line treatment option in patients 65 years and older with chronic lymphocytic leukemia/small lymphocytic leukemia. Blood. 2016;128:234.

8. Brown JR, Hillmen P, O'Brien S, Barrientos JC, Reddy NM, Coutre SE, et al. Extended follow-up and impact of high-risk prognostic factors from the phase 3 RESONATE study in patients with previously treated CLL/SLL. Leukemia. 2018;32:83-91.

9. Furman RR, Sharman JP, Coutre SE, Cheson BD, Pagel JM, Hillmen P, et al. Idelalisib and rituximab in relapsed chronic lymphocytic leukemia. N Engl J Med. 2014;370:997-1007.

10. Stilgenbauer S, Eichhorst B, Schetelig J, Coutre SE, Seymour JF, Munir $\mathrm{T}$, et al. Venetoclax in relapsed or refractory chronic lymphocytic leukaemia with $17 \mathrm{p}$ deletion: a multicentre, openlabel, phase 2 study. Lancet Oncol. 2016;17:768-78.

11. Fischer K, Bahlo J, Fink AM, Goede V, Herling CD, Cramer P, et al. Long-term remissions after FCR chemoimmunotherapy in previously untreated patients with CLL: updated results of the CLL8 trial. Blood. 2016;127:208-15.

12. Eichhorst B, Fink AM, Bahlo J, Busch R, Kovacs G, Maurer C, et al. First-line chemoimmunotherapy with bendamustine and rituximab versus fludarabine, cyclophosphamide, and rituximab in patients with advanced chronic lymphocytic leukaemia (CLL10): an international, open-label, randomised, phase 3, non-inferiority trial. Lancet Oncol. 2016;17:928-42.

13. Michallet AS, Aktan M, Hiddemann W, Ilhan O, Johansson P, Laribi K, et al. Rituximab plus bendamustine or chlorambucil for chronic lymphocytic leukemia: primary analysis of the randomized, open-label MABLE study. Haematologica. 2018;103:698-706.

14. Goede V, Fischer K, Busch R, Engelke A, Eichhorst B, Wendtner $\mathrm{CM}$, et al. Obinutuzumab plus chlorambucil in patients with CLL and coexisting conditions. N Engl J Med. 2014;370:1101-10.

15. Hillmen P, Robak T, Janssens A, Babu KG, Kloczko J, Grosicki $\mathrm{S}$, et al. Chlorambucil plus ofatumumab versus chlorambucil alone in previously untreated patients with chronic lymphocytic leukaemia (COMPLEMENT 1): a randomised, multicentre, openlabel phase 3 trial. Lancet. 2015;385:1873-83.

16. Wierda WG, Zelenetz AD, Gordon LI, Abramson JS, Advani RH, Andreadis CB, et al. NCCN guidelines insights: chronic lymphocytic leukemia/small lymphocytic leukemia, version 1.2017. J Natl Compr Canc Netw. 2017;15:293-311.

17. Goede V, Fischer K, Busch R, Jaeger U, Dilhuydy MS, Wickham $\mathrm{N}$, et al. Chemoimmunotherapy with GA101 plus chlorambucil in patients with chronic lymphocytic leukemia and comorbidity: results of the CLL11 (BO21004) safety run-in. Leukemia. 2013;27:1172-4.

18. Goede V, Fischer K, Engelke A, Schlag R, Lepretre S, Montero $\mathrm{LF}$, et al. Obinutuzumab as frontline treatment of chronic lymphocytic leukemia: updated results of the CLL11 study. Leukemia. 2015;29:1602-4.

19. Goede V, Fischer K, Dyer MJS, Müller L, Smolej L, Di Bernardo $\mathrm{MC}$ et al. Overall survival benefit of obinutuzumab over rituximab when combined with chlorambucil in patients with chronic lymphocytic leukemia and comorbidities: final survival analysis of the CLL11 study. 2017. Abstract S151. https://library.ehaweb.org/eha/ 2018/stockholm/215923/valentin.goede.overall.survival.benefit.of. obinutuzumab.over.rituximab.when.html.

20. Hallek M, Fischer K, Fingerle-Rowson G, Fink AM, Busch R, Mayer J, et al. Addition of rituximab to fludarabine and cyclophosphamide in patients with chronic lymphocytic leukaemia: a randomised, open-label, phase 3 trial. Lancet. 2010;376:1164-74.

21. Skarbnik AP, Faderl S. The role of combined fludarabine, cyclophosphamide and rituximab chemoimmunotherapy in chronic lymphocytic leukemia: current evidence and controversies. Ther Adv Hematol. 2017;8:99-105.

22. Nunes AA, da Silva AS, Souza KM, Koury Cde N, de Mello LM. Rituximab, fludarabine, and cyclophosphamide versus fludarabine and cyclophosphamide for treatment of chronic lymphocytic 
leukemia: A systematic review with meta-analysis. Crit Rev Oncol Hematol. 2015;94:261-9.

23. Rossi D, Terzi-di-Bergamo L, De Paoli L, Cerri M, Ghilardi G, Chiarenza A, et al. Molecular prediction of durable remission after first-line fludarabine-cyclophosphamide-rituximab in chronic lymphocytic leukemia. Blood. 2015;126:1921-4.

24. Böttcher S, Ritgen M, Fischer K, Stilgenbauer S, Busch RM, Fingerle-Rowson G, et al. Minimal residual disease quantification is an independent predictor of progression-free and overall survival in chronic lymphocytic leukemia: a multivariate analysis from the randomized GCLLSG CLL8 trial. J Clin Oncol. 2012;30:980-8.

25. Böttcher S, Hallek M, Ritgen M, Kneba M. The role of minimal residual disease measurements in the therapy for CLL: is it ready for prime time? Hematol Oncol Clin North Am. 2013;27:267-88.

26. Kovacs G, Robrecht S, Fink AM, Bahlo J, Cramer P, von Tresckow J, et al. Minimal residual disease assessment improves prediction of outcome in patients with chronic lymphocytic leukemia (CLL) who achieve partial response: comprehensive analysis of two phase III studies of the German CLL study group. J Clin Oncol. 2016;34:3758-65.

27. Moreton P, Kennedy B, Lucas G, Leach M, Rassam SM, Haynes A, et al. Eradication of minimal residual disease in B-cell chronic lymphocytic leukemia after alemtuzumab therapy is associated with prolonged survival. J Clin Oncol. 2005;23:2971-9.

28. Strati P, Keating MJ, O'Brien SM, Burger J, Ferrajoli A, Jain N, et al. Eradication of bone marrow minimal residual disease may prompt early treatment discontinuation in CLL. Blood. 2014;123:3727-32.

29. Kwok M, Rawstron AC, Varghese A, Evans PA, O'Connor SJ, Doughty C, et al. Minimal residual disease is an independent predictor for 10-year survival in CLL. Blood. 2016;128:2770-3.

30. Wierda WG. Minimal residual disease provides treatment focus for next chronic lymphocytic leukemia advances. J Clin Oncol. 2016;34:3722-3.

31. Santacruz R, Villamor N, Aymerich M, Martinez-Trillos A, López C, Navarro A, et al. The prognostic impact of minimal residual disease in patients with chronic lymphocytic leukemia requiring first-line therapy. Haematologica. 2014;99:873-80.

32. Shanafelt TD, Wang V, Kay NE, Hanson CA, O'Brien SM, Barrientos JC et al. Ibrutinib-Rituximab or Chemoimmunotherapy for Chronic Lymphocytic Leukemia. N Engl J Med. 2019;381:432-43. https://doi.org/10.1056/NEJMoa1817073.

33. Tam CS, O'Brien S, Wierda W, Kantarjian H, Wen S, Do KA, et al. Long-term results of the fludarabine, cyclophosphamide, and rituximab regimen as initial therapy of chronic lymphocytic leukemia. Blood. 2008;112:975-80.

34. Chai-Adisaksopha C, Brown JR. FCR achieves long-term durable remissions in patients with IGHV-mutated CLL. Blood. 2017;130:2278-82.

35. Thompson PA, Tam CS, O'Brien SM, Wierda WG, Stingo F, Plunkett W, et al. Fludarabine, cyclophosphamide, and rituximab treatment achieves long-term disease-free survival in IGHVmutated chronic lymphocytic leukemia. Blood. 2016;127:303-9.

36. Stilgenbauer S, Schnaiter A, Paschka P, Zenz T, Rossi M, Döhner K, et al. Gene mutations and treatment outcome in chronic lymphocytic leukemia: results from the CLL8 trial. Blood. 2014;123:3247-54.
37. Cartron G, de Guibert S, Dilhuydy MS, Morschhauser F, Leblond V, Dupuis J, et al. Obinutuzumab (GA101) in relapsed/refractory chronic lymphocytic leukemia: final data from the phase $1 / 2$ GAUGUIN study. Blood. 2014;124:2196-202.

38. Byrd JC, Flynn JM, Kipps TJ, Boxer M, Kolibaba KS, Carlile DJ, et al. Randomized phase 2 study of obinutuzumab monotherapy in symptomatic, previously untreated chronic lymphocytic leukemia. Blood. 2016;127:79-86.

39. Brown JR, O'Brien S, Kingsley CD, Eradat H, Pagel JM, Lymph $\mathrm{J}$, et al. Obinutuzumab plus fludarabine/cyclophosphamide or bendamustine in the initial therapy of CLL patients: the phase $1 \mathrm{~b}$ GALTON trial. Blood. 2015;125:2779-85.

40. Stilgenbauer S, Leblond V, Foà R, Böttcher S, Ilhan O, Knauf W, et al. Obinutuzumab plus bendamustine in previously untreated patients with CLL: a subgroup analysis of the GREEN study. Leukemia. 2018;32:1778-86.

41. Leblond V, Aktan M, Ferra Coll CM, Dartigeas C, Kisro J, Montillo M, et al. Safety of obinutuzumab alone or combined with chemotherapy for previously untreated or relapsed/refractory chronic lymphocytic leukemia in the Phase 3b GREEN study. Haematologica. 2018;103:1889-98.

42. Rawstron AC, Villamor N, Ritgen M, Böttcher S, Ghia P, Zehnder $\mathrm{JL}$, et al. International standardized approach for flow cytometric residual disease monitoring in chronic lymphocytic leukaemia. Leukemia. 2007;21:956-64.

43. Kalina T, Flores-Montero J, van der Velden VH, Martin-Ayuso M, Böttcher S, Ritgen M, et al. EuroFlow standardization of flow cytometer instrument settings and immunophenotyping protocols. Leukemia. 2012;26:1986-2010.

44. Freeman CL, Morschhauser F, Sehn L, Dixon M, Houghton R, Lamy T, et al. Cytokine release in patients with CLL treated with obinutuzumab and possible relationship with infusion-related reactions. Blood. 2015;126:2646-9.

45. Owen C, Christofides A, Johnson N, Lawrence T, MacDonald D, Ward C. Use of minimal residual disease assessment in the treatment of chronic lymphocytic leukemia. Leuk Lymphoma. 2017;58:2777-85.

46. Shanafelt TD, Borah BJ, Finnes HD, Chaffee KG, Ding W, Leis $\mathrm{JF}$, et al. Impact of ibrutinib and idelalisib on the pharmaceutical cost of treating chronic lymphocytic leukemia at the individual and societal levels. J Oncol Pr. 2015;11:252-8.

47. Chen Q, Jain N, Ayer T, Weirda WG, Flowers CR, O'Brien SM, et al. Economic burden of chronic lymphocytic leukemia in the era of oral targeted therapies in the United States. J Clin Oncol. 2017;35:166-74.

48. Mato AR, Roeker LE, Allan JN, Pagel JM, Brander DM, Hill BT, et al. Outcomes of front-line ibrutinib treated CLL patients excluded from landmark clinical trial. Am J Hematol. 2018;93:1394-401.

49. Mato AR, Nabhan C, Thompson MC, Lamanna N, Brander DM, Hill B et al. Toxicities and outcomes of 616 ibrutinib-treated patients in the United States: a real-world analysis. Haematologica. 2018;103:874-9.

50. Jain N TP, Burger JA, Ferrajoli A, Borthakur G, Bose P, Estrov Z et al. Ibrutinib, fludarabine, cyclophosphamide, and obinutuzumab (iFCG) for firstline treatment of patients with CLL with mutated IGHV and without TP53 aberrations. Blood 2018:132:695. https:// doi.org/10.1182/blood-2018-185. 